\title{
An Objective Approach to Schizophrenia Recognition Utilizing an Adaptive Neuro-Fuzzy Inference (ANFIS) Model
}

\author{
Amadin, F. $\mathbf{I}^{1}$ and Obi, J.C. ${ }^{2}$ \\ Department Of Computer Science, University Of Benin, Benin City. Nigeria. \\ 1frankamadin@uniben.edu; 2tripplejo2k2@yahoo.com
}

\begin{abstract}
Schizophrenia is a brain disorder that distorts the way a person thinks, acts, expresses emotions, perceives reality, and relates to others. A systematic approach and an overview perception has been carried out over the years by different researchers, but none has sufficiently introduced and Adaptive Neuro-Fuzzy Inference System (ANFIS) approach for these prediction which has served as the focal aim of this research paper using available parameters and datasets. Matric Laboratory (MATLAB) 7.0 served as the tool of implementation highlighting various views. The ANFIS training was successful completed at epoch 34, and had an error of 2.47367e-005 and the test error which was generated by the ANFIS was 0.0002511 . The training was accomplished using a constant membership function type at an error tolerance at 0.05 .
\end{abstract}

Keyword: Schizophrenia, ANFIS, Membership Function, Training and Testing Errors

\section{Introduction}

Schizoaffective disorder is a serious mental illness that has features of two different conditions, schizophrenia, and an affective (mood) disorder that may be diagnosed as either major depression or bipolar disorder (WebMD, 2014).

Schizophrenia is a brain disorder that distorts the way a person thinks, acts, expresses emotions, perceives reality, and relates to others. Depression is an illness that is marked by feelings of sadness, worthlessness, or hopelessness, as well as problems concentrating and remembering details (WebMD, 2014). Bipolar disorder is characterized by cycling mood changes, including severe highs (mania) and lows (depression).

Schizoaffective disorder is a lifelong illness that can impact all areas of daily living, including work or school, social contacts, and relationships. Most people with this illness have periodic episodes, called relapses, when their symptoms surface. While there is no cure for schizoaffective disorder, symptoms often can be controlled with proper treatment (WebMD, 2014).

While the exact cause of schizoaffective disorder is not known, researchers believe that genetic, biochemical, and environmental factors are involved (Help Guide, 2014 WebMD, 2014 and Right Diagnosis, 2014:

- Genetics (heredity): A tendency to develop schizoaffective disorder may be passed on from parents to their children. 
Amadin, F. I and Obi, J.C; An Objective Approach to Schizophrenia Recognition Utilizing an Adaptive Neuro-Fuzzy Inference (ANFIS) Model, Transactions on Machine Learning and Artificial Intelligence, Volume 3 No 2 April, (2015); pp: 1-9

- Brain chemistry: People with schizophrenia and mood disorders may have abnormalities in the functioning of brain circuits that regulate mood and thinking.

- Environmental factors: Theories suggest that certain environmental factors such as a viral infection, poor social interactions or highly stressful situations may trigger schizoaffective disorder in people who have inherited a tendency to develop the disorder. However, the relationships between biological and environmental factors that may lead to schizoaffective disorder are not well understood.

The positive symptoms of Schizophrenia are delusion, hallucination, disorganised speech and thinking affect and catatonic behaviour the negative while the negative symptoms associated with schizophrenia occur as a result of degeneration of everyday activity which might include Flattened Affect, Alogia and Avolition also known as loss of motivation from the medical point of view, the person might show lack or disinterest in socializing (Mayoclinic, 2014 and webMd, 2014). This is as a result of degeneration in catatonic behaviour. Both positive and negative symptoms of Schizophrenia has their functionalities; if the patients displays only positive symptoms then the patient might be suffering from acute schizophrenia whereas the chronic schizophrenia occur when patients displays signs of negative symptoms (HelpGuide, 2014).

According to the Diagnostic and Statistical Manual of Mental Disorder IV (DSMMD-IV) for a patient to be diagnosed with schizophrenia both positive and negative characteristic signs and symptoms (both positive and negative) must be present for a significant period (a month) with the symptoms delusion and hallucination persisting for at least 6 months. Schizophrenia can be classified under five categories they are (MedicineNet, 2014, RightDiagnosis, 2014 and WebMD, 2014):

- Paranoid Schizophrenia: The patient is obsessed with someone following him/her, spicing on him/her or tricking him/her. It might even involve symptoms like hallucinations but muddle behaviour of loss of speech is not evident here.

- Disorganized Schizophrenia: this form of schizophrenia involves positive symptoms like disorganized speech and behaviour which might also show loss of self-expression.

- Catatonic Schizophrenia: Patient suffering from catatonic schizophrenia might exhibit severe bewilderment in behaviour

- Undifferentiated Schizophrenia: A patient suffering from undifferentiated schizophrenia might show signs of delusions, hallucinations, disorganized speech or behaviour, catatonic behaviour or negative symptoms.

- Residual Schizophrenia: A patients suffering from residual schizophrenia must show signs of only negative symptoms.

\section{Review of Related Literature}

Several research works has focused on Schizoprehnia,

Dwight et al., (2010), carried out a research on comparison of cognitive structure in schizophrenia patients and healthy controls using confirmatory factor analysis based on the underlining evidence that cognitive task performance breaks down into the same broad domains in schizophrenia. In reaching a conclusive boundary, a confirmatory factor analysis (CFA) to compare the latent structure of a broad 
neuropsychological battery in schizophrenia patients $(n=148)$ and healthy controls $(n=157)$ were utilized. Conclusively it was agreed that CFA possesses higher efficiency.

Tara et al., (2013) focused on suicide prevention in schizophrenia spectrum disorders and psychosis embedding a systematic approach using Cochrane, PubMed and PsyclNFO databases dataset as methodology. Conclusively, it was agreed that Psychosocial interventions may be effective in reducing suicidal behaviour in patients with schizophrenia spectrum disorders and psychosis, although the additional benefit of these interventions above that contributed by a control condition or treatment-asusual is not clear.

Other research work includes (Víctor and Manuel, 2003; Steffen and Todd, 2007, Olivier and Marc, 2005).

From the exhaustive literature review, an Objective approach has rear been adopted for the diagnosis of schizophrenia, based on this demerit; An Objective Approach to Schizophrenia Recognition Utilizing an Adaptive Neuro-Fuzzy Inference (ANFIS) Model was inspired.

\section{Methodology: Adaptive Neuro Fuzzy Inference System (ANFIS) Architecture}

The Adaptive Neuro Fuzzy System is one of the several types of Neuro-fuzzy system that combines both the learning capabilities of the Neuro fuzzy system and the explanatory power of the fuzzy inference system. It has 6 layers and each layer comprises of neurons that performs specific function. The fuzzy inference system can be built using the mamandi or the sugeno-tagaki model. The ANFIS uses the sugeno-tagaki model in building the fuzzy inference system.

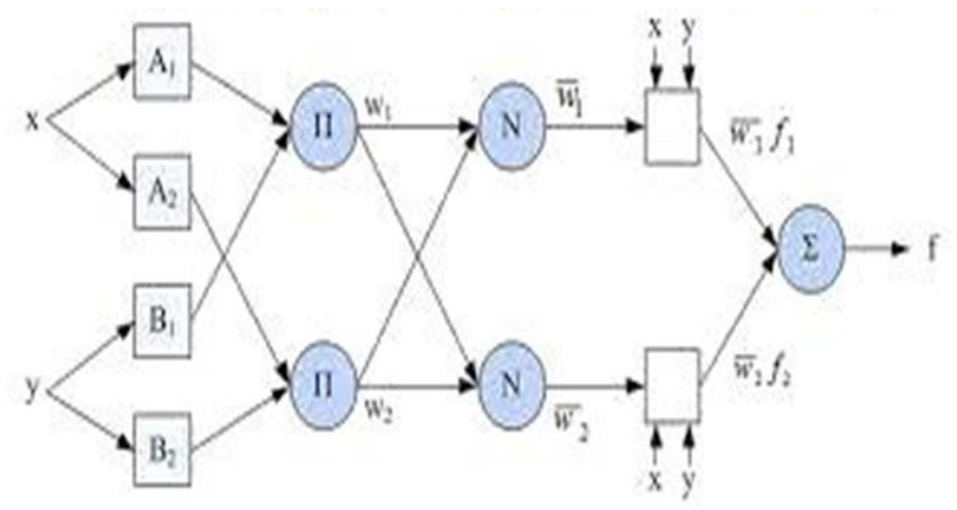

Figure 2: Adaptive Neuro-Fuzzy Inference System (ANFIS) Model for Schizophrenia Application

a) Layer 1: (Input Layer): This is the first layer it is called the input layer.

b) Layer 2: (Fuzzification layer): The second layer of the Adaptive Neuro Fuzzy Inference System (ANFIS) model is called fuzzification layer. It could also be called the membership function layer. In this layer the input coming in from the input layer is mapped to fuzzy set using the bell membership function.

c) Layer 3: (Rule Layer): The third layer in the ANFIS is called the rule layer. The rules in this layer are built using the sugeno rule fuzzy model. Each neuron in this layer receives the input from the fuzzification layer and computes the output. 


$$
O_{2, i}=w_{i}=\mu_{A_{i}}(x) \times \mu_{B_{i}}(x) \quad i=1,2
$$

d) Layer 4 (Normalization layer): The fourth layer of the ANFIS is called the normalization layer. The neurons in this layer receive inputs from the rule layer and calculate the normalization firing strength. This is sent to the fifth layer.

$$
O_{3, i}=\bar{w}_{i}=\frac{w_{i}}{w_{1}+w_{2}} \quad i=1,2
$$

a) Layer 5 (Defuzzification layer): The fifth layer in the Adaptive-Neuro Fuzzy System is called the defuzzification layer. The neurons in this layer receive it's input from the fourth layer.

$$
O_{4, i}=\bar{w}_{i} f_{i}=\bar{w}_{i}\left(p_{i} x+q_{i} y+r_{i}\right)
$$

b) Layer 6 (Output Layer): the sixth layer of the Adaptive Neuro Fuzzy Inference System is called the output layer. It give the overall out of the Adaptive Neuro Fuzzy System. It contains a single neuron that performs summation of all the incoming inputs from the fifth layer.

$$
O_{5,1}=\text { overall output }=\sum_{i} \bar{w}_{i} f_{i}
$$

\section{Stimulations and Results}

The dataset used for the computer stimulation was retrieved from eight-two case sample was randomly selected from the sample and it contained $76 \%$ schizophrenic cases and $24 \%$ free cases. $40 \%$ of this data was randomly selected and used to train the system at a cutoff $0.05 \%$ the data was trained for 34 epochs while $30 \%$ of the dataset was used for testing and $12 \%$ was used for checking. The stimulation result is as follows.

Table 1: Membership Function, Training Error and Test Error Representation

\begin{tabular}{|l|l|l|l|}
\hline S/N & Membership Function & Training Error & Test Error \\
\hline 1. & Bell-curve Membership Function & $2.47367 e-005$ & 0.0002511 \\
\hline 2. & Triangular Membership Function & 0.0082133 & 0.0075454 \\
\hline 3. & Trapezoidal Membership Function & 0.0097643 & 0.0087654 \\
\hline 4. & Guass1 Membership Function & 0.0054332 & 0.005143 \\
\hline 5. & Guass2 Membership Function & 0.0074532 & 0.005432 \\
\hline
\end{tabular}

The results were gotten using linear membership function an at an error tolerance level of 0.05 


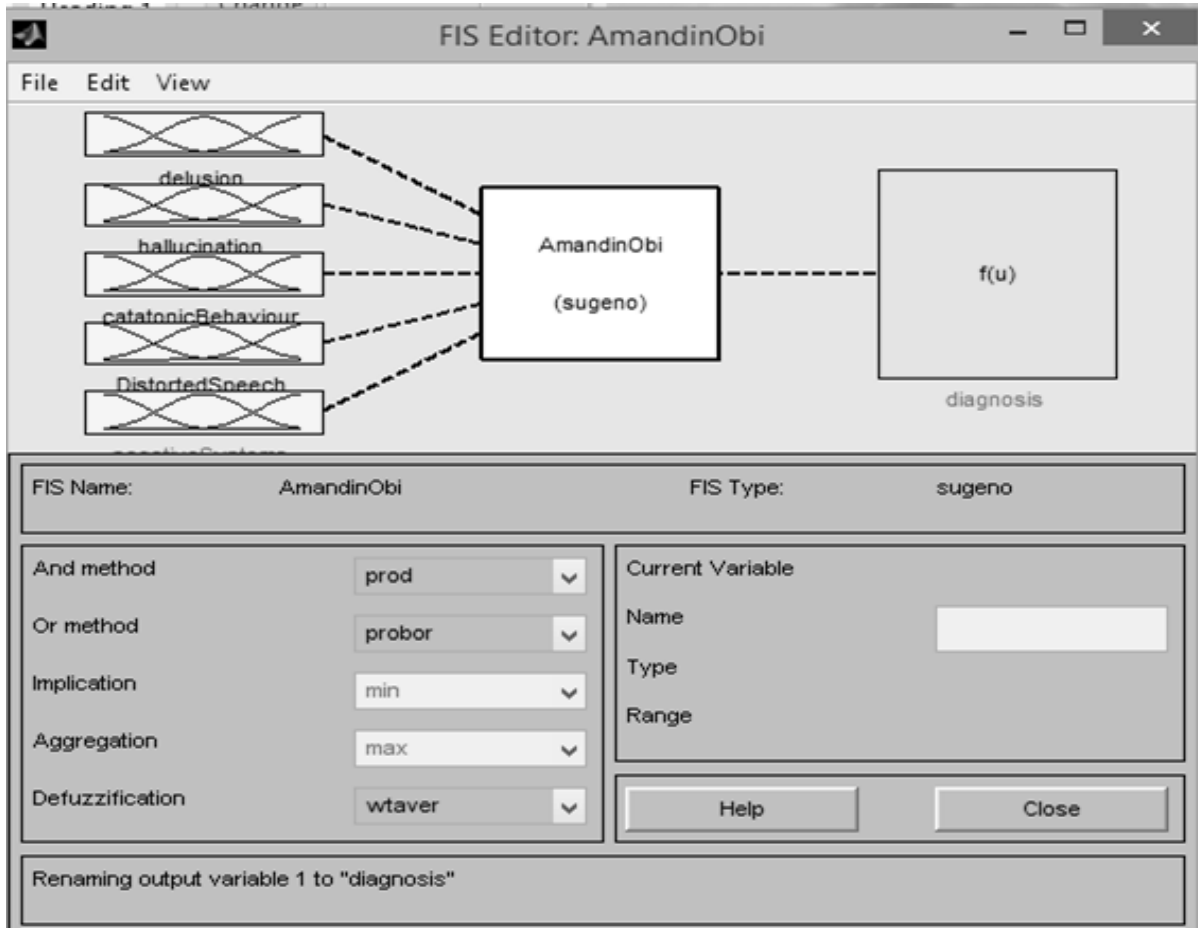

Figure 2: ANFIS Text View Editor

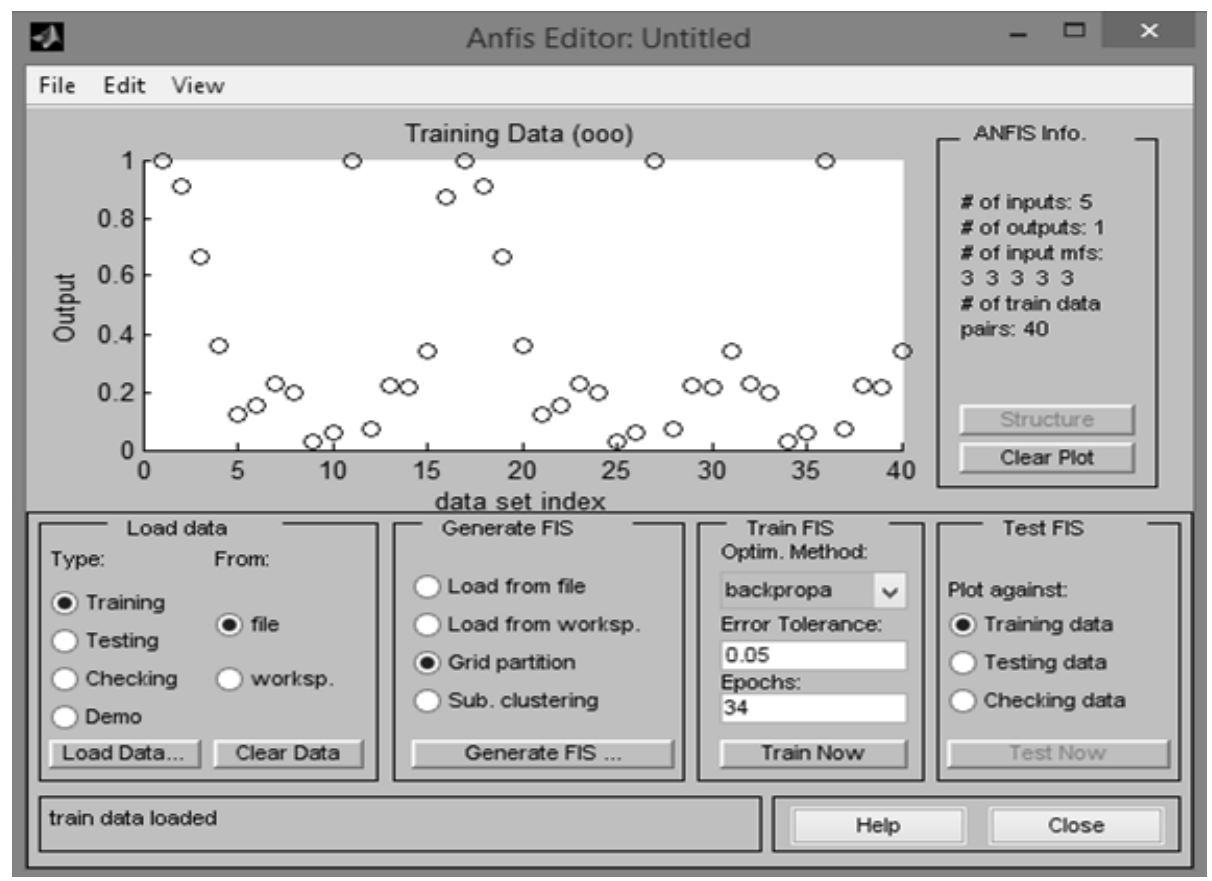

Figure 3: ANFIS Training Data Editor 
Amadin, F. I and Obi, J.C; An Objective Approach to Schizophrenia Recognition Utilizing an Adaptive Neuro-Fuzzy Inference (ANFIS) Model, Transactions on Machine Learning and Artificial Intelligence, Volume 3 No 2 April, (2015); pp: $1-9$

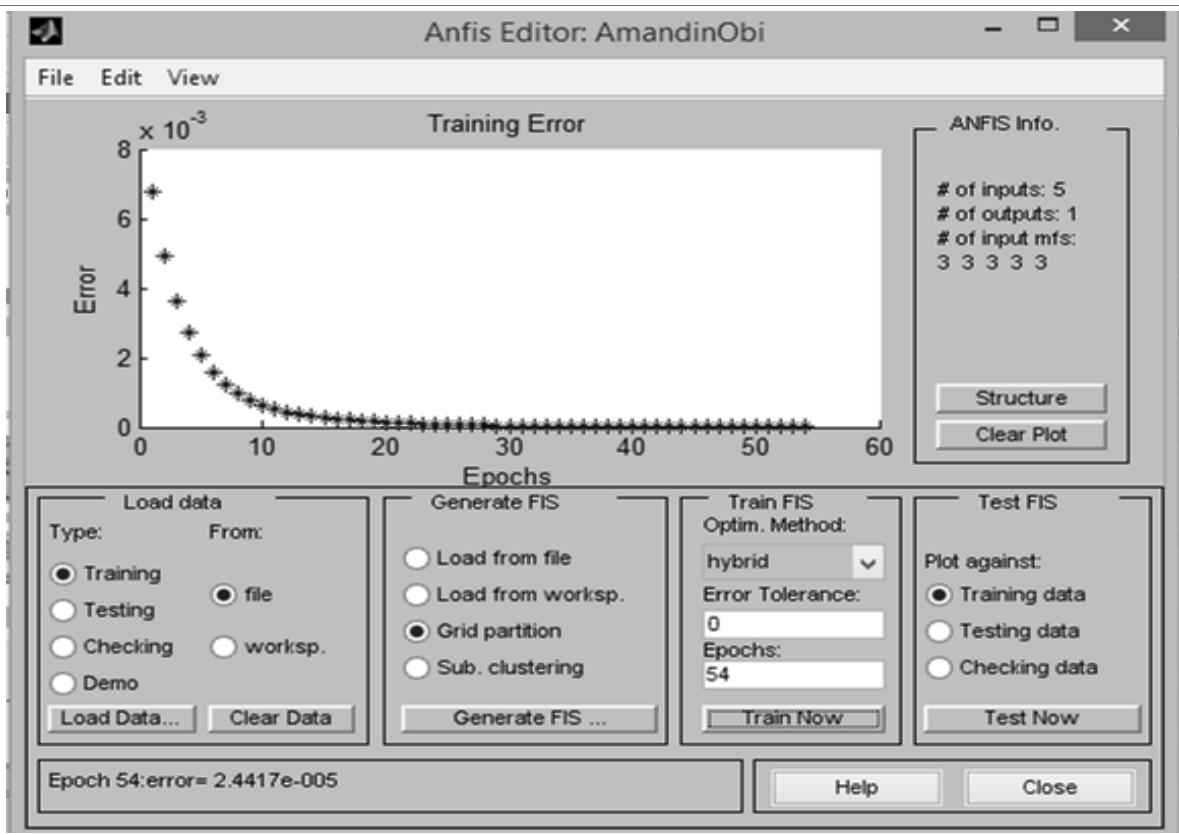

Figure 4: ANFIS Training Error Editor

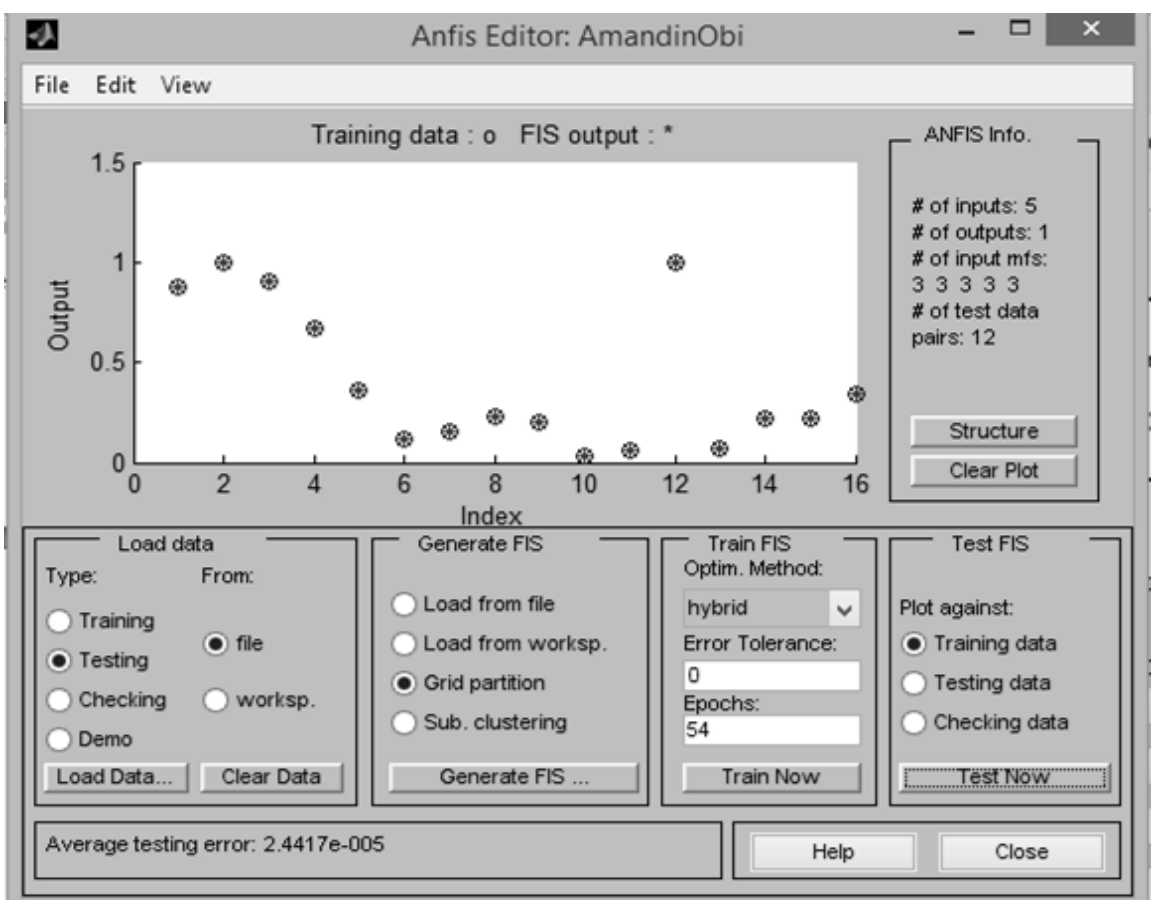

Figure 5: ANFIS Output Editor 


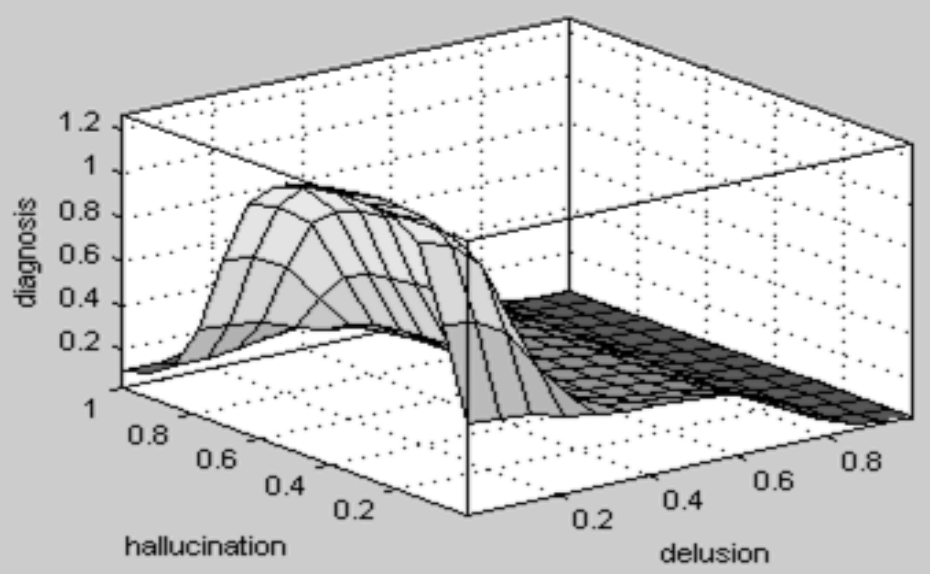

Figure 6: Surface View Illustrating Hallucination and Delusion

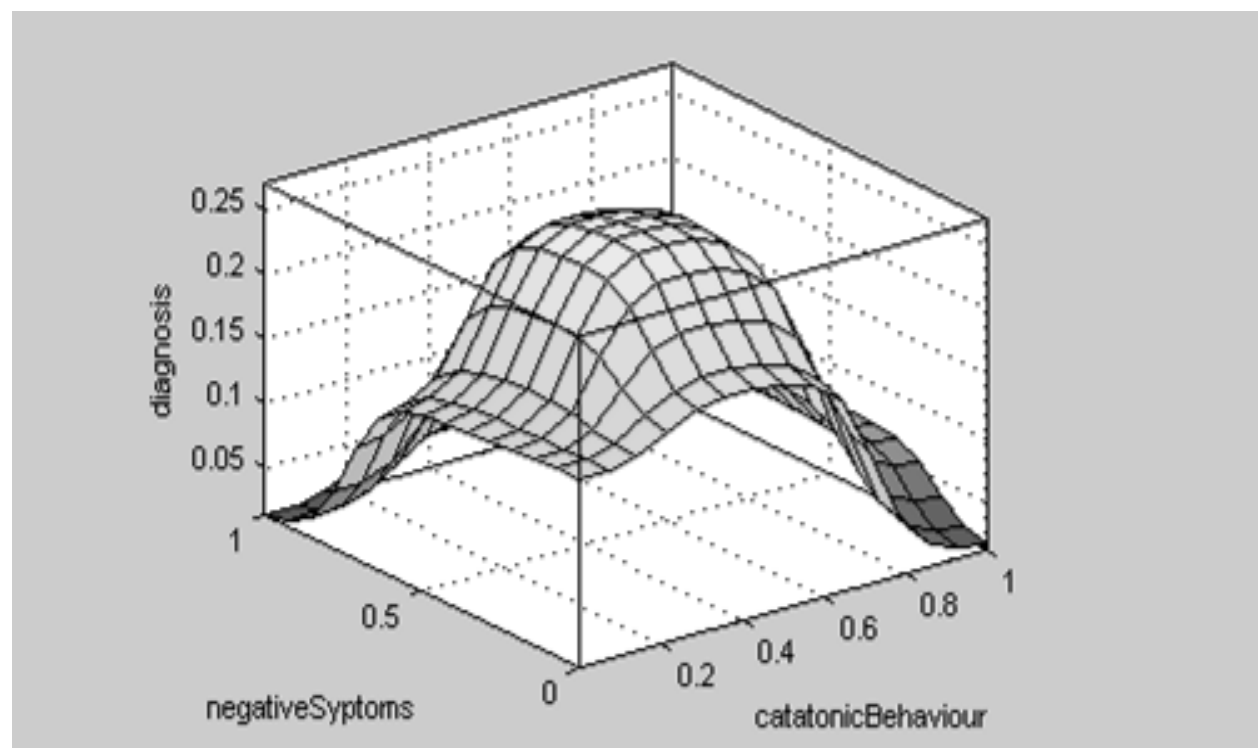

Figure 7: Surface View Illustrating Hallucination and Delusion 


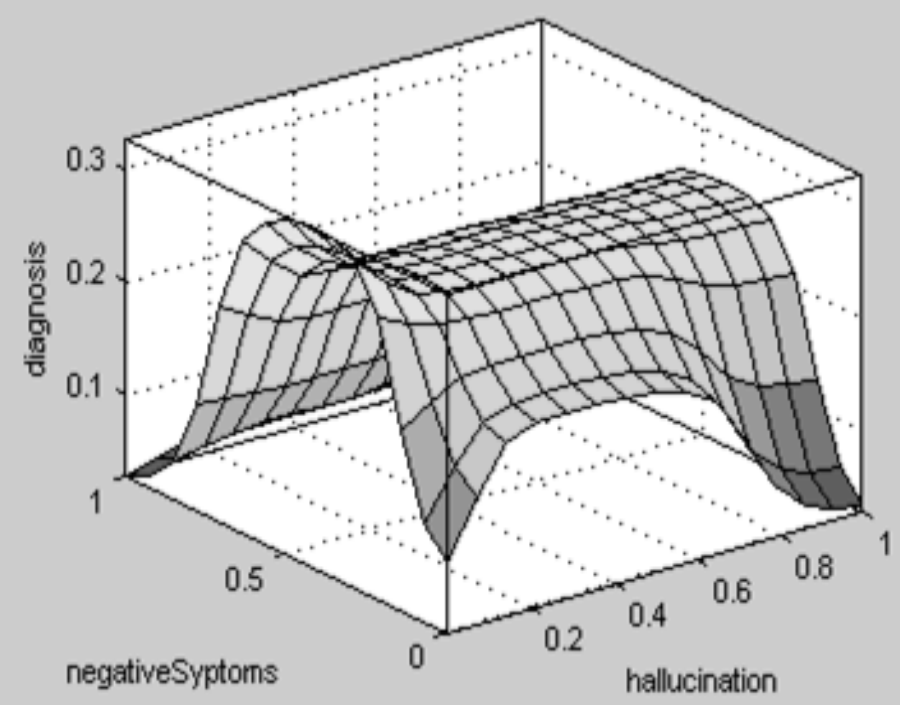

Figure 8: Surface View Illustrating Negative Symptoms and Hallucination

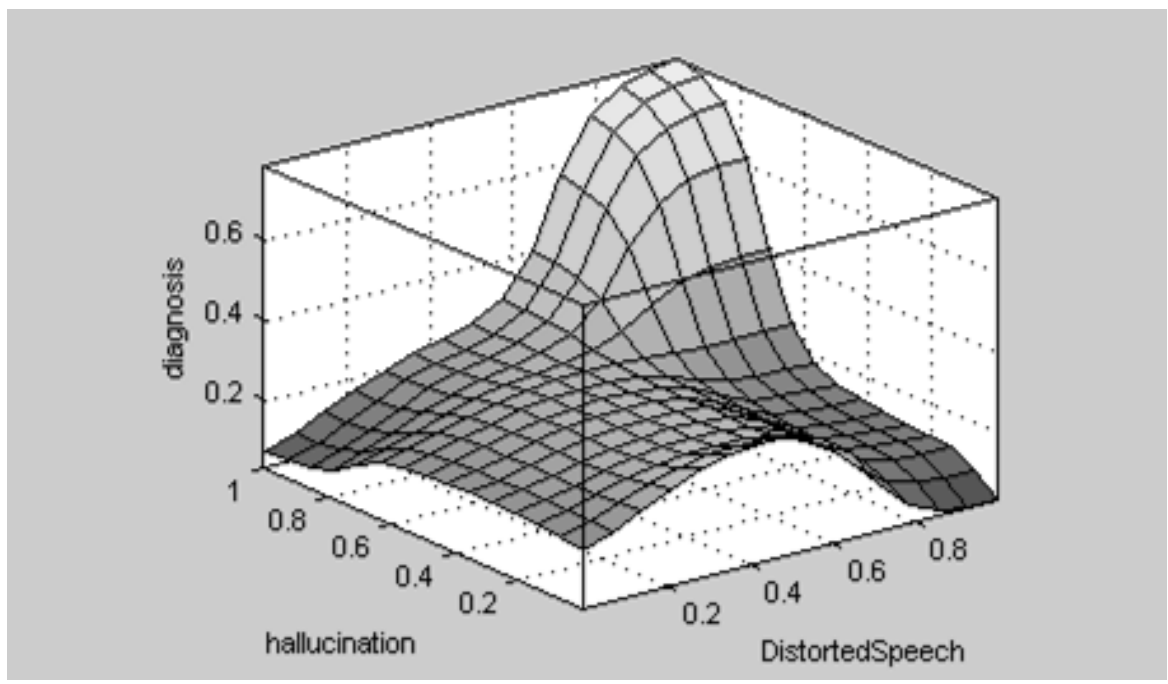

Figure 9: Surface View Illustrating Hallucination and Distorted Speech

\section{Conclusion}

To draw the inference from the experiment it has been clearly shown that the bell curve membership function shows the least training error when used in training the dataset. The training was completed at 34 epochs and had an error of 2.47367e-005 and the test error which was generated by the ANFIS was 0.0002511 . The training was accomplished using a constant membership function type at an error tolerance at 0.05 . 


\section{REFERENCE}

[1] Dwight D., Daniel J. R., Monica E. C., James M. G. and Ruben C. G., (2006), A comparison of cognitive structure in schizophrenia patients and healthy controls using confirmatory factor analysis, Schizophrenia Research 85 (2006) 20 - 29, Available on ScienceDirect.com

[2] Help Guide, (2014), Schizophrenia" retrieved online from HelpGuide.com

[3] Mayo clinic (2014), "Schizophrenia and Treatment" retrieved online from http://www.mayoclinic.org/diseasesconditions/schizophrenia/basics/symptoms/con20021077

[4] Medicine NET (2014), "Schizophrenia", Retrieved online MedicineNET.Com

[5] NIMH: National Institute for Mental Health (2014), "Schizophrenia" retrieved online www.nimh.nih.gov/health/topics/schizophrenia/

[6] Olivier G. and Marc L. (2005), Neurobiology of Dopamine in Schizophrenia, Departments of Psychiatry and Radiology Columbia University College of Physicians and Surgeons, New York, NY

[7] Right Diagnosis (2014), "Schizophrenia" retrieved online from RightDiagnosis.com

[8] Steffen M. and Todd S. W. (2007), Metacognitive training in schizophrenia: from basic research to knowledge translation and intervention, Curr Opin Psychiatry 20:619-625, Wolters Kluwer Health | Lippincott Williams \& Wilkins.

[9] Tara D., Alison C., Janie B. G., Bregje V. S., Katherine F., Kanupriya K. H. Pim C.

[10] and Helen C (2013), Suicide prevention in schizophrenia spectrum disorders and psychosis: a systematic review Donkeret al. BMC Psychology 2013,1:6 retrieved online from http://biomedcentral.com/

[11] Víctor P. and Manuel J.C. (2003), The Diagnosis of Schizophrenia: Old Wine in New Bottles, International Journal of Psychology and Psychological Therapy 2003, Vol. 3, № 2, pp. 141-152

[12] WebMD (2014), "Schizophrenia: Diagnosis and Treatment" retrieved online from WebMD.com 